\title{
Asymptotic profile of solutions to the semilinear beam equation
}

Yunpeng Zhang ${ }^{1 *}$ and Yanling $\mathrm{Li}^{2}$

${ }^{\text {*Correspondence: }}$ ypzhangep@yeah.net

${ }^{1}$ College of Electric Power, North China University of Water Resources and Electric Power, Zhengzhou, 450011, China

Full list of author information is available at the end of the article

\begin{abstract}
In this paper, we investigate the initial value problem for the semilinear beam equation. Under a small condition on the initial value, we prove the global existence and optimal decay estimate of solutions. Moreover, we show that as time tends to infinity, the solution is asymptotic to a diffusion wave, which is given explicitly in terms of the solution of parabolic equation.
\end{abstract}

MSC: 35L30; 35L 75

Keywords: beam equation; decay estimate; asymptotic profile; diffusion wave

\section{Introduction}

We investigate the initial value problem for the following semilinear beam equation:

$$
u_{t t}-\alpha u_{x x}+u_{t}+u_{x x x x}=f\left(u_{x}\right)_{x}
$$

with the initial value

$$
t=0: \quad u=u_{0}(x), \quad u_{t}=u_{1}(x) .
$$

Here $u=u(x, t)$ is the unknown function of $x \in \mathbb{R}$ and $t>0, b>0$, and $\alpha>0$ are constants. The nonlinear term $f(v)$ is a given smooth function of $v \in \mathbb{R}$. More precisely,

$$
f(v)=f(0)+f^{\prime}(0) v+g(v)
$$

where $g(v)=O\left(v^{2}\right)$ for $v \rightarrow 0$.

This initial value problem was studied by $[1,2]$ when $f$ satisfies

$$
\left\{\begin{array}{l}
|f(v)| \leq C|v|^{p}, \quad\left|f^{\prime}(v)\right| \leq C|v|^{p-1} \\
\left|f\left(v_{1}\right)-f\left(v_{2}\right)\right| \leq C\left(\left|v_{1}\right|^{p-1}+\left|v_{2}\right|^{p-1}\right)\left|v_{1}-v_{2}\right| \\
\left|f^{\prime}\left(v_{1}\right)-f^{\prime}\left(v_{2}\right)\right| \leq C\left(\left|v_{1}\right|^{p-2}+\left|v_{2}\right|^{p-2}\right)\left|v_{1}-v_{2}\right|
\end{array}\right.
$$

with $p \geq 2$, so that $f^{\prime}(0)=0$. Here $C$ is independent of $v, v_{1}$, and $v_{2}$. [1] proved that there exists a global solution $u \in C\left([0, \infty) ; H^{2} \cap L^{1}\right)$ to the problem (1.1), (1.2) under smallness condition on the initial data $u_{0} \in W^{2,1} \cap H^{2}$ and $u_{1} \in L^{2} \cap L^{1}$. In particular, they showed the decay estimates:

$$
\|u\|_{L^{q}} \leq C(1+t)^{-\frac{1}{2}\left(1-\frac{1}{q}\right)}, \quad 1 \leq q \leq \infty
$$


and

$$
\left\|\partial_{x}^{2} u\right\|_{L^{q}} \leq C(1+t)^{-\frac{1}{2}\left(1-\frac{1}{q}\right)-1}, \quad 2 \leq q \leq \infty
$$

In addition to the above assumptions, suppose that the initial data $u_{0}, u_{1} \in L_{1}^{1}$, Takeda and Yoshikawa [2] established the following asymptotic profile of global solution:

$$
\left\|u(t)-M G_{\alpha}(t)-(\tilde{M}+N) \partial_{x} G_{\alpha}(t)\right\|_{L^{q}} \leq C(1+t)^{-\frac{1}{2}\left(1-\frac{1}{q}\right)-\frac{1}{2}}, \quad 1 \leq q \leq \infty,
$$

where

$$
M=\int_{-\infty}^{\infty}\left(u_{0}+u_{1}\right)(x) d x, \quad G_{\alpha}(x, t)=F^{-1}\left[e^{-\alpha \xi^{2} t}\right], \quad \tilde{M}=-\int_{-\infty}^{\infty} x\left(u_{0}+u_{1}\right)(x) d x
$$

and

$$
N=\int_{0}^{\infty} \int_{-\infty}^{\infty} f\left(\partial_{x} u(x, t)\right) d x d t
$$

The main purpose of our present paper is two-fold: first, we try to recover all the results about global existence and decay estimate of solution in Takeda and Yoshikawa [1] under some assumptions on the initial data $\left(u_{0}(x), u_{1}(x)\right)$ and the nonlinear function $f$, which is much weaker than those needed in Takeda and Yoshikawa's arguments. More precisely, the condition $u_{0}(x) \in W^{2,1} \cap H^{2}, u_{1}(x) \in L^{2} \cap L^{1}$ and (1.3) have been relaxed to $u_{0}(x) \in$ $H^{s+2} \cap L^{1}, u_{1}(x) \in H^{s} \cap L^{1}(s \geq 0)$ and the nonlinear function $f$ satisfies $f^{\prime}(0)>-\alpha$ in this paper. Second, we show that the solution is asymptotic to a diffusion wave, given explicitly in terms of the solution of parabolic equation that is different from the one in [2]. For the details, we refer to Theorem 4.1. Moreover, under some additional assumptions on the initial data, we also prove that the convergence rates of our new asymptotic profile are better than that obtained by [2]. For details, we refer to Theorem 4.2.

The study of the global existence and asymptotic behavior of solutions to hyperbolictype equations has a long history. We refer to [3, 4] for hyperbolic equations, [5-7] for the damped wave equation and [8-16] for various aspects of dissipation of the plate equation.

The paper is organized as follows. In Section 2, we study the decay property of the solution to the linear problem. Then, in Section 3, we prove the global existence and decay estimate of the solutions. Finally, we prove that the solution is asymptotic to a diffusion wave, which is given explicitly in terms of the solution of the parabolic equation in Section 4 .

Notations We give some notations which are used in this paper. Let $\mathcal{F}[u]$ denote the Fourier transform of $u$ defined by

$$
\hat{u}(\xi)=\mathcal{F}[u]=\int_{\mathbb{R}^{n}} e^{-i \xi \cdot x} u(x) d x,
$$

and we denote its inverse transform by $\mathcal{F}^{-1}$.

For $1 \leq p \leq \infty, L^{p}=L^{p}\left(\mathbb{R}^{n}\right)$ denotes the usual Lebesgue space with the norm $\|\cdot\|_{L^{p}}$. The usual Sobolev space of $s$ is defined by $W^{s, p}=\left(I-\partial_{x}^{2}\right)^{-\frac{s}{2}} L^{p}$ with the norm $\|f\|_{W^{s, p}}=$ $\left\|\left(I-\partial_{x}^{2}\right)^{\frac{s}{2}} f\right\|_{L^{p}}$. 
Finally, in this paper, we denote every positive constant by the same symbol $C$ or $c$ which will not lead to any confusion. [.] is the Gauss symbol.

\section{Linear problem}

\subsection{Solution formula}

The aim of this subsection is to derive the solution formula for the problem (1.1), (1.2). We first investigate the linearized equation of (1.1):

$$
u_{t t}-\beta u_{x x}+u_{t}+u_{x x x x}=0,
$$

with the initial data in (1.2), where $\beta=\alpha+f^{\prime}(0)>0$. We apply the Fourier transform to (2.1). This yields

$$
\hat{u}_{t t}+\xi^{2} \hat{u}_{t}+\left(\beta \xi^{2}+\xi^{4}\right) \hat{u}=0 .
$$

The corresponding initial values are given as

$$
t=0: \quad \hat{u}=\hat{u}_{0}(\xi), \quad \hat{u}_{t}=\hat{u}_{1}(\xi) .
$$

The characteristic equation of $(2.2)$ is

$$
\lambda^{2}+\lambda+\beta \xi^{2}+\xi^{4}=0
$$

Let $\lambda=\lambda_{ \pm}(\xi)$ be the corresponding eigenvalues of (2.4), we obtain

$$
\lambda_{ \pm}(\xi)=\frac{-1 \pm \sqrt{1-4 \xi^{2}\left(\beta+\xi^{2}\right)}}{2} .
$$

The solution to the problem (2.2), (2.3) in the Fourier space is then given explicitly in the form

$$
\hat{u}(\xi, t)=\hat{G}(\xi, t)\left(\hat{u}_{1}(\xi)+\hat{u}_{0}(\xi)\right)+\hat{H}(\xi, t) \hat{u}_{0}(\xi),
$$

where

$$
\hat{G}(\xi, t)=\frac{1}{\lambda_{+}(\xi)-\lambda_{-}(\xi)}\left(e^{\lambda_{+}(\xi) t}-e^{\lambda_{-}(\xi) t}\right)
$$

and

$$
\hat{H}(\xi, t)=\frac{1}{\lambda_{+}(\xi)-\lambda_{-}(\xi)}\left(\left(\lambda_{+}(\xi)+1\right) e^{\lambda_{-}(\xi) t}-\left(\lambda_{-}(\xi)+1\right) e^{\lambda_{+}(\xi) t}\right) .
$$

We define $G(x, t)$ and $H(x, t)$ by

$$
G(x, t)=\mathcal{F}^{-1}[\hat{G}(\xi, t)](x)
$$

and

$$
H(x, t)=\mathcal{F}^{-1}[\hat{H}(\xi, t)](x),
$$


respectively, where $\mathcal{F}^{-1}$ denotes the inverse Fourier transform. Then, applying $\mathcal{F}^{-1}$ to (2.6), we obtain

$$
u(t)=G(t) *\left(u_{1}+u_{0}\right)+H(t) * u_{0} .
$$

By the Duhamel principle, we obtain the solution formula to (1.1), (1.2),

$$
u(t)=G(t) *\left(u_{1}+u_{0}\right)+H(t) * u_{0}+\int_{0}^{t} G(t-\tau) * g\left(u_{x}\right)_{x}(\tau) d \tau
$$

where $g(v)$ is a smooth function; it satisfies $g(v)=O\left(v^{2}\right)$.

\subsection{Decay property}

The aim of this subsection is to establish decay estimates of the solution operators $G(t)$ and $H(t)$ appearing in (2.9) and (2.10), respectively.

Lemma 2.1 The solution of the problem (2.2), (2.3) satisfies

$$
\left|\hat{u}_{t}(\xi, t)\right|^{2}+\left(1+\xi^{2}\right)^{2}|\hat{u}(\xi, t)|^{2} \leq C e^{-c \rho(\xi) t}\left(\left|\hat{u}_{1}(\xi)\right|^{2}+\left(1+\xi^{2}\right)^{2}\left|\hat{u}_{0}(\xi)\right|^{2}\right)
$$

for $\xi \in \mathbb{R}$ and $t \geq 0$, where $\rho(\xi)=\frac{\xi^{2}}{1+\xi^{2}}$.

Proof Multiplying (2.2) by $\overline{\hat{u}}_{t}$ and taking the real part yields

$$
\frac{1}{2} \frac{d}{d t}\left\{\left|\hat{u}_{t}\right|^{2}+\beta \xi^{2}|\hat{u}|^{2}+\xi^{4}|\hat{u}|^{2}\right\}+\left|\hat{u}_{t}\right|^{2}=0 .
$$

Multiplying (2.2) by $\overline{\hat{u}}$ and taking the real part, we obtain

$$
\frac{1}{2} \frac{d}{d t}\left\{|\hat{u}|^{2}+2 \operatorname{Re}\left(\hat{u}_{t} \overline{\hat{u}}\right)\right\}+\beta \xi^{2}|\hat{u}|^{2}+\xi^{4}|\hat{u}|^{2}-\left|\hat{u}_{t}\right|^{2}=0 .
$$

Multiplying both sides of (2.14) by 2 and summing up the resulting equation and (2.15) yields

$$
\frac{d}{d t} E+F=0
$$

where

$$
E=\left|\hat{u}_{t}\right|^{2}+\left(\frac{1}{2}+\beta \xi^{2}+\xi^{4}\right)|\hat{u}|^{2}+\operatorname{Re}\left(\hat{u}_{t} \overline{\hat{u}}\right)
$$

and

$$
F=\left|\hat{u}_{t}\right|^{2}+\beta \xi^{2}|\hat{u}|^{2}+\xi^{4}|\hat{u}|^{2} .
$$

A simple computation implies that

$$
C E_{0} \leq E \leq C E_{0},
$$


where

$$
E_{0}=\left|\hat{u}_{t}\right|^{2}+\left(1+\xi^{2}\right)^{2}|\hat{u}|^{2} .
$$

Note that

$$
F \geq c \rho(\xi) E_{0} .
$$

It follows from (2.17) that

$$
F \geq c \rho(\xi) E
$$

Using (2.16) and (2.18), we get

$$
\frac{d}{d t} E+c \rho(\xi) E \leq 0
$$

Thus

$$
E(\xi, t) \leq e^{-c \rho(\xi) t} E(\xi, 0)
$$

which together with (2.17) proves the desired estimates (2.13). Then we have completed the proof of the lemma.

Lemma 2.2 Let $\hat{G}(\xi, t)$ and $\hat{H}(\xi, t)$ be the fundamental solution of $(2.1)$ in the Fourier space, which are given in (2.7) and (2.8), respectively. Then we have the estimates

$$
\left|\hat{G}_{t}(\xi, t)\right|^{2}+\left(1+\xi^{2}\right)^{2}|\hat{G}(\xi, t)|^{2} \leq C e^{-c \rho(\xi) t}
$$

and

$$
\left|\hat{H}_{t}(\xi, t)\right|^{2}+\left(1+\xi^{2}\right)^{2}|\hat{H}(\xi, t)|^{2} \leq C\left(1+\xi^{2}\right)^{2} e^{-c \rho(\xi) t}
$$

for $\xi \in \mathbb{R}$ and $t \geq 0$, where $\rho(\xi)=\frac{\xi^{2}}{1+\xi^{2}}$.

Proof Firstly, we investigate the problem (2.1), (1.2) with $\hat{u}_{0}(\xi)=0$, from (2.6), we obtain

$$
\hat{u}(\xi, t)=\hat{G}(\xi, t) \hat{u}_{1}(\xi), \quad \hat{u}_{t}(\xi, t)=\hat{G}_{t}(\xi, t) \hat{u}_{1}(\xi) .
$$

Substituting the equalities into (3.1) with $\hat{u}_{0}(\xi)=0$, we get (2.19).

In what follows, we consider the problem (2.1), (1.2) with $\hat{u}_{1}(\xi)=0$; it follows from (2.6) that

$$
\hat{u}(\xi, t)=(\hat{G}+\hat{H})(\xi, t) \hat{u}_{0}(\xi), \quad \hat{u}_{t}(\xi, t)=\left(\hat{G}_{t}+\hat{H}_{t}\right)(\xi, t) \hat{u}_{0}(\xi) .
$$

Substituting the equalities into (2.13) with $\hat{u}_{1}(\xi)=0$, we get the desired estimate $(2.20)$. The lemma is proved. 
Let

$$
G_{0}(x, t)=F^{-1}\left[e^{-\beta \xi^{2} t}\right](x)=\frac{1}{\sqrt{4 \pi \beta t}} e^{e^{-\frac{x^{2}}{4 \beta t}}}
$$

be the fundamental solution to $u_{t}-\beta u_{x x}=0$.

Lemma 2.3 Let $\hat{G}(\xi, t)$ and $\hat{H}(\xi, t)$ be the fundamental solution of $(2.1)$ in the Fourier space, which are given in (2.6) and (2.7), respectively. Then there is a small positive number $R_{0}$ such that if $|\xi| \leq R_{0}$ and $t \geq 0$, we have the following estimates:

$$
\begin{aligned}
& \left|\left(\hat{G}-\hat{G}_{0}\right)(\xi, t)\right|+|\hat{H}(\xi, t)| \leq C \xi^{2} e^{-c \xi^{2} t}+C e^{-c t}, \\
& \left|\hat{G}_{t}(\xi, t)\right| \leq C \xi^{2} e^{-c \xi^{2} t}+C e^{-c t}
\end{aligned}
$$

and

$$
\left|\partial_{t}\left(\hat{G}-\hat{G}_{0}\right)(\xi, t)\right|+\left|\hat{H}_{t}(\xi, t)\right| \leq C \xi^{4} e^{-c \xi^{2} t}+C e^{-c t} .
$$

Proof For sufficiently small $\xi$, using the Taylor formula, we get

$$
\begin{aligned}
& \lambda_{+}(\xi)=-\beta \xi^{2}+O\left(\xi^{4}\right), \quad \lambda_{-}(\xi)=-1+O\left(\xi^{2}\right), \\
& \frac{1}{\lambda_{+}(\xi)-\lambda_{-}(\xi)}=1+2 \beta \xi^{2}+O\left(\xi^{4}\right) .
\end{aligned}
$$

We rewrite $\hat{G}(\xi, t)$ in $(2.6)$ as

$$
\begin{aligned}
\hat{G}(\xi, t)= & e^{-\beta \xi^{2} t}+\left(\frac{1}{\lambda_{+}(\xi)-\lambda_{-}(\xi)}-1\right) e^{\lambda_{+}(\xi) t} \\
& +\left(e^{\left(\lambda_{+}(\xi)+\beta \xi^{2}\right) t}-1\right) e^{-\beta \xi^{2} t}-\frac{e^{\lambda_{-}(\xi) t}}{\lambda_{+}(\xi)-\lambda_{-}(\xi)} .
\end{aligned}
$$

For sufficiently small $\xi$, from (2.24) and (2.25), we immediately obtain

$$
\left|\left(\hat{G}-\hat{G}_{0}\right)(\xi, t)\right| \leq C \xi^{2} e^{-c \xi^{2} t}+C e^{-c t} .
$$

For sufficiently small $\xi$, from (2.7) and (2.24), we immediately get

$$
|\hat{H}(\xi, t)| \leq C \xi^{2} e^{-c \xi^{2} t}+C e^{-c t}
$$

Thus we get (2.21). The other estimates are proved similarly and we omit the details. The proof of Lemma 2.3 is completed.

Lemma 2.4 Let $G(x, t)$ and $H(x, t)$ be the fundamental solutions of $(2.1)$, which are given in (2.9) and (2.10), respectively. Let $1 \leq p \leq 2$, and let $k, j$, and $l$ be nonnegative integers. Then we have

$$
\left\|\partial_{x}^{k} G(t) * \phi\right\|_{L^{2}} \leq C(1+t)^{-\frac{1}{2}\left(\frac{1}{p}-\frac{1}{2}\right)-\frac{k-j}{2}}\left\|\partial_{x}^{j} \phi\right\|_{L^{p}}+C e^{-c t}\left\|\partial_{x}^{k+l-2} \phi\right\|_{L^{2}},
$$




$$
\left\|\partial_{x}^{k} H(t) * \phi\right\|_{L^{2}} \leq C(1+t)^{-\frac{1}{2}\left(\frac{1}{p}-\frac{1}{2}\right)-\frac{k+2-j}{2}}\left\|\partial_{x}^{j} \phi\right\|_{L^{p}}+C e^{-c t}\left\|\partial_{x}^{k+l} \phi\right\|_{L^{2}}
$$

for $0 \leq j \leq k$, where $k+l-2 \geq 0$ in (2.26). Similarly, we have

$$
\begin{aligned}
& \left\|\partial_{x}^{k} G_{t}(t) * \phi\right\|_{L^{2}} \leq C(1+t)^{-\frac{1}{2}\left(\frac{1}{p}-\frac{1}{2}\right)-\frac{k+2-j}{2}}\left\|\partial_{x}^{j} \phi\right\|_{L^{p}}+C e^{-c t}\left\|\partial_{x}^{k+l} \phi\right\|_{L^{2}} \\
& \left\|\partial_{x}^{k} H_{t}(t) * \phi\right\|_{L^{2}} \leq C(1+t)^{-\frac{1}{2}\left(\frac{1}{p}-\frac{1}{2}\right)-\frac{k+4-j}{2}}\left\|\partial_{x}^{j} \phi\right\|_{L^{p}}+C e^{-c t}\left\|\partial_{x}^{k+l+2} \phi\right\|_{L^{2}}
\end{aligned}
$$

for $0 \leq j \leq k+2$ in (2.28) and $0 \leq j \leq k+4$ in (2.29).

Proof We only prove (2.26). By the Plancherel theorem and (2.19), the Hausdorff-Young inequality, we obtain

$$
\begin{aligned}
\left\|\partial_{x}^{k} G(t) * \phi\right\|_{L^{2}}^{2} & =\int_{|\xi| \leq 1}|\xi|^{2 k}|\hat{G}(\xi, t)|^{2}|\hat{\phi}(\xi)|^{2} d \xi+\int_{|\xi| \geq 1}|\xi|^{2 k}|\hat{G}(\xi, t)|^{2}|\hat{\phi}(\xi)|^{2} d \xi \\
& \leq C \int_{|\xi| \leq 1}|\xi|^{2 k} e^{-c \xi^{2} t}|\hat{\phi}(\xi)|^{2} d \xi+C e^{-c t} \int_{|\xi| \geq 1}|\xi|^{2 k}\left(1+|\xi|^{2}\right)^{-2}|\hat{\phi}(\xi)|^{2} d \xi \\
& =: \mathcal{A}_{1}+\mathcal{A}_{2} .
\end{aligned}
$$

For the term $\mathcal{A}_{1}$, letting $\frac{1}{p^{\prime}}+\frac{1}{p}=1$, we have

$$
\begin{aligned}
\mathcal{A}_{1} & \leq C \int_{|\xi| \leq 1}|\xi|^{2 k-2} e^{-c \xi^{2} t}|\hat{\phi}(\xi)|^{2} d \xi \\
& \leq C\left\||\xi|^{j} \hat{\phi}\right\|_{L^{p^{\prime}}}^{2}\left(\int_{|\xi| \leq 1}|\xi|^{2(k-j) p} e^{-c q \xi^{2} t} d \xi\right)^{\frac{1}{p}} \\
& \leq C(1+t)^{-\left(\frac{1}{p}-\frac{1}{2}\right)-(k-j)}\left\|\partial_{x}^{j} \phi\right\|_{L^{p}}^{2},
\end{aligned}
$$

where we used the Hölder inequality with $\frac{2}{p^{\prime}}+\frac{1}{q}=1$ and the Hausdorff-Young inequality $\|\hat{v}\|_{L^{p^{\prime}}} \leq C\|v\|_{L^{p}}$ for $v=\partial_{x}^{j} \phi$. On the other hand, we can estimate the term $\mathcal{A}_{2}$ simply as

$$
\begin{aligned}
\mathcal{A}_{2} & \leq C e^{-c t} \int_{|\xi| \geq 1}|\xi|^{2 k-4}|\hat{\phi}(\xi)|^{2} d \xi \\
& \leq C e^{-c t} \int_{|\xi| \geq 1}|\xi|^{2(k+l-2)}|\hat{\phi}(\xi)|^{2} d \xi \\
& \leq C e^{-c t}\left\|\partial_{x}^{k+l-2} \phi\right\|_{L^{2}}^{2},
\end{aligned}
$$

where $k+l-2 \geq 0$.

Combining the above three inequalities yields (2.26). This completes the proof of Lemma 2.4.

From Lemma 2.4, we immediately have the following corollary.

Corollary 2.1 Let $G(x, t)$ and $H(x, t)$ be the fundamental solution of $(2.1)$, which are given in (2.8) and (2.9), respectively. Let $1 \leq p \leq 2$, and let $k$, $j$, and $l$ be nonnegative integers. Then we have

$$
\left\|\partial_{x}^{k} G(t) * g_{x}\right\|_{L^{2}} \leq C(1+t)^{-\frac{1}{2}\left(\frac{1}{p}-\frac{1}{2}\right)-\frac{k+1-j}{2}}\left\|\partial_{x}^{j} g\right\|_{L^{p}}+C e^{-c t}\left\|\partial_{x}^{k+l-1} g\right\|_{L^{2}}
$$


for $0 \leq j \leq k+1$ and $k+l-1 \geq 0$. Also we have

$$
\left\|\partial_{x}^{k} G_{t}(t) * g_{x}\right\|_{L^{2}} \leq C(1+t)^{-\frac{1}{2}\left(\frac{1}{p}-\frac{1}{2}\right)-\frac{k+3-j}{2}}\left\|\partial_{x}^{j} g\right\|_{L^{p}}+C e^{-c t}\left\|\partial_{x}^{k+l+1} g\right\|_{L^{2}}
$$

for $0 \leq j \leq k+3$.

Lemma 2.5 Let $G(x, t)$ be the fundamental solution of (2.1), given in (2.8) and let $G_{0}(x, t)$ be the fundamental solution of (2.1), given in (2.8). Let $1 \leq p \leq 2$, and let $k, j$, and $l$ be nonnegative integers. Then we have

$$
\left\|\partial_{x}^{k}\left(G-G_{0}\right)(t) * \phi\right\|_{L^{2}} \leq C(1+t)^{-\frac{1}{2}\left(\frac{1}{p}-\frac{1}{2}\right)-\frac{k+2-j}{2}}\left\|\partial_{x}^{j} \phi\right\|_{L^{p}}+C e^{-c t}\left\|\partial_{x}^{k+l} \phi\right\|_{L^{2}}
$$

for $0 \leq j \leq k+2$. Similarly,

$$
\left\|\partial_{x}^{k}\left(G-G_{0}\right)_{t}(t) * \phi\right\|_{L^{2}} \leq C(1+t)^{-\frac{1}{2}\left(\frac{1}{p}-\frac{1}{2}\right)-\frac{k+4-j}{2}}\left\|\partial_{x}^{j} \phi\right\|_{L^{p}}+C e^{-c t}\left\|\partial_{x}^{k+l+2} \phi\right\|_{L^{2}}
$$

for $0 \leq j \leq k+4$.

Proof The proof of Lemma 2.5 is similar to the proof of Lemma 2.4. By employing (2.21) and (2.23), we can prove Lemma 2.5. We omit the details.

\section{Global existence and asymptotic behavior of solutions to (1.1), (1.2)}

The purpose of this section is to prove global existence and optimal decay estimate of solutions to the initial value problem (1.1), (1.2). We need the following lemma, which comes from [17] (see also [18]).

Lemma 3.1 Assume that $f=f(v)$ is a smooth function. Suppose that $f(v)=O\left(|v|^{1+\theta}\right)(\theta \geq 1$ is an integer) when $|v| \leq v_{0}$. Then for integer $m \geq 0$, if $v \in W^{m, q}\left(\mathbb{R}^{n}\right) \cap L^{p}\left(\mathbb{R}^{n}\right) \cap L^{\infty}\left(\mathbb{R}^{n}\right)$ and $\|v\|_{L^{\infty}} \leq v_{0}$, the following inequalities hold:

$$
\left\|\partial_{x}^{m} f(v)\right\|_{L^{r}} \leq C\|v\|_{L^{p}}\left\|\partial_{x}^{m} v\right\|_{L^{q}}\|v\|_{L^{\infty}}^{\theta-1}
$$

where $\frac{1}{r}=\frac{1}{p}+\frac{1}{q}, 1 \leq p, q, r \leq+\infty$.

Theorem 3.1 Let $s \geq 0$. Suppose that $f(v)$ is a smooth function and $f^{\prime}(0)>-\alpha$. Assume that $u_{0} \in H^{s+2} \cap L^{1}, u_{1} \in H^{s} \cap L^{1}$. Put

$$
E_{0}:=\left\|u_{0}\right\|_{H^{s+2} \cap L^{1}}+\left\|u_{1}\right\|_{H^{s} \cap L^{1}} .
$$

Then there exists a positive constant $\delta_{1}$ such that if $E_{0} \leq \delta_{0}$, and the initial value problem (1.1), (1.2) has a unique global solution $u(x, t)$ satisfying

$$
u \in C^{0}\left([0, \infty) ; H^{s+2}(\mathbb{R})\right) \cap C^{1}\left([0, \infty) ; H^{s}(\mathbb{R})\right) .
$$

Moreover, the solution satisfies the decay estimate

$$
\left\|\partial_{x}^{k} u(t)\right\|_{L^{2}} \leq C E_{0}(1+t)^{-\frac{1}{4}-\frac{k}{2}}
$$


and

$$
\left\|\partial_{x}^{k} u_{t}(t)\right\|_{L^{2}} \leq C E_{0}(1+t)^{-\frac{5}{4}-\frac{k}{2}}
$$

where $0 \leq k \leq s+2$ in (3.2) and $0 \leq k \leq \sin (3.3)$.

Proof The existence and uniqueness of small solutions can be proved by the contraction mapping principle. Here we only show the decay estimates (3.2) and (3.3) for the solution $u$ of (2.12) satisfying $\|u(t)\|_{L^{\infty}} \leq M_{0}$ with some $M_{0}$. To this end, we introduce the quantity

$$
X(t)=\sum_{k=0}^{s+2} \sup _{0 \leq \tau \leq t}(1+\tau)^{\frac{1}{4}+\frac{k}{2}}\left\|\partial_{x}^{k} u(\tau)\right\|_{L^{2}} .
$$

Here we note that

$$
\left\|u_{x}(t)\right\|_{L^{\infty}} \leq C X(t)(1+t)^{-1}
$$

provided that $s \geq 0$. This follows from the Gagliardo-Nirenberg inequality, $\left\|u_{x}\right\|_{L^{\infty}} \leq$ $C\left\|u_{x x}\right\|_{L^{2}}^{\frac{1}{2}}\left\|u_{x}\right\|_{L^{2}}^{\frac{1}{2}}$ and the definition of $X(t)$ in (3.4).

Applying $\partial_{x}^{k}$ to (2.12) and taking the $L^{2}$ norm, we obtain

$$
\begin{aligned}
\left\|\partial_{x}^{k} u(t)\right\|_{L^{2}} \leq & \left\|\partial_{x}^{k} G(t) *\left(u_{0}+u_{1}\right)\right\|_{L^{2}}+\left\|\partial_{x}^{k} H(t) * u_{0}\right\|_{L^{2}} \\
& +\int_{0}^{t}\left\|G(t-\tau) * \partial_{x} g\left(u_{x}(\tau)\right)\right\|_{L^{2}} d \tau \\
=: & I_{1}+I_{2}+J .
\end{aligned}
$$

Firstly, we estimate $I_{1}$. We apply (2.26) with $p=1, j=0$ and $l=0(l=2$ for $k=0, l=1$ for $k=1)$. This yields

$$
I_{1} \leq C(1+t)^{-\frac{1}{4}-\frac{k}{2}}\left\|u_{0}+u_{1}\right\|_{L^{1}}+C e^{-c t}\left\|\partial_{x}^{(k-2)_{+}}\left(u_{0}+u_{1}\right)\right\|_{L^{2}} \leq C E_{0}(1+t)^{-\frac{1}{4}-\frac{k}{2}},
$$

where $(k-2)_{+}=\max \{k-2,0\}$. Similarly, applying (2.27) with $p=1, j=0$, and $l=0$ to the term $I_{2}$, we have

$$
I_{2} \leq C(1+t)^{-\frac{1}{4}-\frac{k}{2}}\left\|u_{0}\right\|_{L^{1}}+C e^{-c t}\left\|\partial_{x}^{k} u_{0}\right\|_{L^{2}} \leq C E_{0}(1+t)^{-\frac{1}{4}-\frac{k}{2}}
$$

We estimate the nonlinear term $J$. We divide $J$ into two parts and write $J=J_{1}+J_{2}$, where $J_{1}$ and $J_{2}$ are corresponding to the time intervals $[0, t / 2]$ and $[t / 2, t]$, respectively. For the term $J_{1}$, we apply (2.30) with $p=1, j=0$, and $l=0$. This yields

$$
\begin{aligned}
J_{1} \leq & C \int_{0}^{t / 2}(1+t-\tau)^{-\frac{3}{4}-\frac{k}{2}}\left\|g\left(u_{x}\right)(\tau)\right\|_{L^{1}} d \tau \\
& +C \int_{0}^{t / 2} e^{-c(t-\tau)}\left\|\partial_{x}^{(k-1)_{+}} g\left(u_{x}\right)(\tau)\right\|_{L^{2}} d \tau \\
= & J_{11}+J_{12} .
\end{aligned}
$$


Here we see that $\left\|g\left(u_{x}\right)\right\|_{L^{1}} \leq C\left\|u_{x}\right\|_{L^{2}}^{2}$ by Lemma 3.1. Thus we have $\left\|g\left(u_{x}\right)(\tau)\right\|_{L^{1}} \leq$ $C X(t)^{2}(1+\tau)^{-\frac{3}{2}}$. Therefore we can estimate the term $J_{11}$ as

$$
\begin{aligned}
J_{11} & \leq C X(t)^{2} \int_{0}^{t / 2}(1+t-\tau)^{-\frac{3}{4}-\frac{k}{2}}(1+\tau)^{-\frac{3}{2}} d \tau \\
& \leq C X(t)^{2}(1+t)^{-\frac{3}{4}-\frac{k}{2}} \int_{0}^{t / 2}(1+\tau)^{-\frac{3}{2}} d \tau \\
& \leq C X(t)^{2}(1+t)^{-\frac{3}{4}-\frac{k}{2}}
\end{aligned}
$$

On the other hand, we have $\left\|\partial_{x}^{(k-1)_{+}} g\left(u_{x}\right)\right\|_{L^{2}} \leq C\left\|u_{x}\right\|_{L^{\infty}}\left\|\partial_{x}^{k} u\right\|_{L^{2}}$ by Lemma 3.1. Therefore, using (3.5), we find that $\left\|\partial_{x}^{(k-1)_{+}} g\left(u_{x}\right)\right\|_{L^{2}} \leq C X(t)^{2}(1+\tau)^{-\frac{5}{4}-\frac{k}{2}}$. Consequently, we can estimate the term $J_{12}$ as

$$
J_{12} \leq C X(t)^{2} \int_{0}^{t / 2} e^{-c(t-\tau)}(1+\tau)^{-\frac{5}{4}-\frac{k}{2}} d \tau \leq C X(t)^{2} e^{-c t} .
$$

Finally, we estimate the term $J_{2}$ on the time interval $[t / 2, t]$. Applying (2.30) with $p=2$, $j=k$, and $l=0$, and using $\left\|\partial_{x}^{(k-1)_{+}} g\left(u_{x}\right)(\tau)\right\|_{L^{2}} \leq C X(t)^{2}(1+\tau)^{-\frac{5}{4}-\frac{k}{2}}$, we obtain

$$
\begin{aligned}
J_{2} & \leq C \int_{t / 2}^{t}(1+t-\tau)^{-1}\left\|\partial_{x}^{(k-1)_{+}} g\left(u_{x}\right)(\tau)\right\|_{L^{2}} d \tau+C \int_{t / 2}^{t} e^{-c(t-\tau)}\left\|\partial_{x}^{(k-1)_{+}} g\left(u_{x}\right)(\tau)\right\|_{L^{2}} d \tau \\
& \leq C X(t)^{2} \int_{t / 2}^{t}(1+t-\tau)^{-1}(1+\tau)^{-\frac{5}{4}-\frac{k}{2}} d \tau \\
& \leq C X(t)^{2}(1+t)^{-\frac{5}{4}-\frac{k}{2}+\varepsilon}
\end{aligned}
$$

Thus we have shown that

$$
J \leq C X(t)^{2}(1+t)^{-\frac{3}{4}-\frac{k}{2}} .
$$

Substituting all these estimates into (3.6), we obtain

$$
(1+t)^{\frac{1}{4}+\frac{k}{2}}\left\|\partial_{x}^{k} u(t)\right\|_{L^{2}} \leq C E_{0}+C X(t)^{2}
$$

for $0 \leq k \leq s+2$. Consequently, we have $X(t) \leq C E_{0}+C X(t)^{2}$, from which we can deduce $X(t) \leq C E_{0}$, provided that $E_{0}$ is suitably small. This proves the decay estimate (3.2).

In what follows, we prove the decay estimate (3.3) for the time derivative $u_{t}$. For this purpose we differentiate (2.12) with respect to $t$ to obtain

$$
u_{t}(t)=G_{t}(t) *\left(u_{0}+u_{1}\right)+H_{t}(t) * u_{0}+\int_{0}^{t} G_{t}(t-\tau) * \partial_{x} g\left(u_{x}\right)(\tau) d \tau
$$

Applying $\partial_{x}^{k}$ to (3.11) and taking the $L^{2}$ norm, we have

$$
\begin{aligned}
\left\|\partial_{x}^{k} u_{t}(t)\right\|_{L^{2}} \leq & \left\|\partial_{x}^{k} G_{t}(t) *\left(u_{0}+u_{1}\right)\right\|_{L^{2}}+\left\|\partial_{x}^{k} H_{t}(t) * u_{0}\right\|_{L^{2}} \\
& +\int_{0}^{t}\left\|\partial_{x}^{k} G_{t}(t-\tau) * \partial_{x} g\left(u_{x}\right)(\tau)\right\|_{L^{2}} d \tau \\
= & I_{1}^{\prime}+I_{2}^{\prime}+J^{\prime}
\end{aligned}
$$


where $0 \leq k \leq s$. For the term $I_{1}^{\prime}$, we apply (2.28) with $p=1, j=0$, and $l=0$ to get

$$
I_{1}^{\prime} \leq C(1+t)^{-\frac{5}{4}-\frac{k}{2}}\left\|u_{0}+u_{1}\right\|_{L^{1}}+e^{-c t}\left\|\partial_{x}^{k}\left(u_{1}+u_{0}\right)\right\|_{L^{2}} \leq C E_{0}(1+t)^{-\frac{5}{4}-\frac{k}{2}} .
$$

Also, for the term $I_{2}^{\prime}$, applying (2.29) with $p=1, j=0$ and $l=0$, we have

$$
I_{2}^{\prime} \leq C(1+t)^{-\frac{9}{4}-\frac{k}{2}}\left\|u_{0}\right\|_{L^{1}}+e^{-c t}\left\|\partial_{x}^{k+2} u_{0}\right\|_{L^{2}} \leq C E_{0}(1+t)^{-\frac{9}{4}-\frac{k}{2}} .
$$

To estimate the nonlinear term $J^{\prime}$, we rewrite $J^{\prime}=J_{1}^{\prime}+J_{2}^{\prime}$, where $J_{1}^{\prime}$ and $J_{2}^{\prime}$ correspond to the time intervals $[0, t / 2]$ and $[t / 2, t]$, respectively. For the term $J_{1}^{\prime}$, we apply $(2.31)$ with $p=1$, $j=0$, and $l=0$. This yields

$$
\begin{aligned}
J_{1}^{\prime} \leq & C \int_{0}^{t / 2}(1+t-\tau)^{-\frac{7}{4}-\frac{k}{2}}\left\|g\left(u_{x}\right)(\tau)\right\|_{L^{1}} d \tau \\
& +C \int_{0}^{t / 2} e^{-c(t-\tau)}\left\|\partial_{x}^{k+1} g\left(u_{x}\right)(\tau)\right\|_{L^{2}} d \tau \\
= & : J_{11}^{\prime}+J_{12}^{\prime} .
\end{aligned}
$$

Since $\left\|g\left(u_{x}\right)(\tau)\right\|_{L^{1}} \leq C X(t)^{2}(1+\tau)^{-\frac{3}{2}}$ as before, we can estimate the term $J_{11}^{\prime}$ as

$$
\begin{aligned}
J_{11}^{\prime} & \leq C X(t)^{2} \int_{0}^{t / 2}(1+t-\tau)^{-\frac{7}{4}-\frac{k}{2}}(1+\tau)^{-\frac{3}{2}} d \tau \\
& \leq C X(t)^{2}(1+t)^{-\frac{7}{4}-\frac{k}{2}} .
\end{aligned}
$$

Also, the term $J_{12}^{\prime}$ is estimated similarly as before and we have $J_{12}^{\prime} \leq C X(t)^{2} e^{-c t}$. Finally, we estimate the term $J_{2}^{\prime}$ by applying (2.31) with $p=2, j=k+2$, and $l=0$ and obtain

$$
\begin{aligned}
J_{2}^{\prime} & \leq C \int_{t / 2}^{t}(1+t-\tau)^{-1}\left\|\partial_{x}^{k+1} g\left(u_{x}\right)(\tau)\right\|_{L^{2}} d \tau+C \int_{t / 2}^{t} e^{-c(t-\tau)}\left\|\partial_{x}^{k+1} g\left(u_{x}\right)(\tau)\right\|_{L^{2}} d \tau \\
& \leq C X(t)^{2} \int_{t / 2}^{t}(1+t-\tau)^{-1}(1+\tau)^{-\frac{9}{4}-\frac{k}{2}} d \tau \\
& \leq C X(t)^{2}(1+t)^{-\frac{9}{4}-\frac{k}{2}+\varepsilon},
\end{aligned}
$$

where we used the estimate $\left\|\partial_{x}^{k+1} g\left(u_{x}\right)(\tau)\right\|_{L^{2}} \leq C X(t)^{2}(1+\tau)^{-\frac{9}{4}-\frac{k}{2}}$. Consequently we have shown that

$$
J^{\prime} \leq C X(t)^{2}(1+t)^{-\frac{9}{4}-\frac{k}{2}}
$$

Substituting all these estimates together with the previous estimate $X(t) \leq C E_{0}$ into (3.12), we arrive at the desired estimate (3.3) for $0 \leq k \leq s$. This completes the proof of Theorem 3.1.

The above proof of Theorem 3.1 shows that the solution $u$ to the integral equation (2.12) is asymptotic to the linear solution $u_{L}$ given by the formula $u_{L}(t):=G(t) *\left(u_{0}+u_{1}\right)+H(t) *$ $u_{0}$ in (2.11) as $t \rightarrow \infty$. This result is stated as follows. 
Corollary 3.1 Assume the same conditions of Theorem 3.1. Then the solution $u$ of the problem (1.1), (1.2), which is constructed in Theorem 3.1, can be approximated by the solution $u_{L}$ to the linearized problem (2.1), (1.2) as $t \rightarrow \infty$. More precisely, we have the following asymptotic relations:

$$
\begin{aligned}
& \left\|\partial_{x}^{k}\left(u-u_{L}\right)(t)\right\|_{L^{2}} \leq C E_{0}^{2}(1+t)^{-\frac{3}{4}-\frac{k}{2}}, \\
& \left\|\partial_{x}^{k}\left(u-u_{L}\right)_{t}(t)\right\|_{L^{2}} \leq C E_{0}^{2}(1+t)^{-\frac{7}{4}-\frac{k}{2}}
\end{aligned}
$$

for $0 \leq k \leq s+2$ and $0 \leq k \leq s$, respectively, where $u_{L}(t):=G(t) *\left(u_{0}+u_{1}\right)+H(t) * u_{0}$ is the linear solution.

\section{Asymptotic profile}

In this section, our aim is to establish an asymptotic profile to our global solution that is constructed in Theorem 4.1. In the previous section, we have shown that the solution $u$ to the problem (1.1), (1.2) can be approximated by the linear solution $u_{L}$. In what follows, we shall derive a simpler asymptotic profile of the linear solution $u_{L}$.

Let $v$ be the solution to the initial data problem

$$
v_{t}-\beta v_{x x}=0, \quad t=0: \quad v=\left(u_{0}+u_{1}\right)(x) .
$$

Then

$$
v=G_{0}(x, t) *\left(u_{0}+u_{1}\right)(x)
$$

gives a asymptotic profile of the linear solution $u_{L}$. In fact we have the following.

Lemma 4.1 Let $s \geq 0$. Assume that $u_{0}, u_{1} \in H^{s+2} \cap L^{1}$ and put $E_{0}=\left\|\left(u_{0}, u_{1}\right)\right\|_{H^{s+2} \cap L^{1}}$. Let $u_{L}$ be the linear solution and let $v$ be defined by (4.2). Then we have

$$
\left\|\partial_{x}^{k}\left(u_{L}-v\right)(t)\right\|_{L^{2}} \leq C E_{0}(1+t)^{-\frac{5}{4}-\frac{k}{2}}
$$

for $0 \leq k \leq s+2$.

Proof Note that $\left(u_{L}-v\right)(t)=\left(G-G_{0}\right)(t) *\left(u_{0}+u_{1}\right)+H(t) * u_{0}$, so for the proof of (4.3), it suffices to show the following estimates:

$$
\begin{aligned}
& \left\|\partial_{x}^{k}\left(G-G_{0}\right)(t) * \phi\right\|_{L^{2}} \leq C(1+t)^{-\frac{5}{4}}\|\phi\|_{L^{1}}+C e^{-c t}\left\|\partial_{x}^{k} \phi\right\|_{L^{2}}, \\
& \left\|\partial_{x}^{k} H(t) * \phi\right\|_{L^{2}} \leq C(1+t)^{-\frac{5}{4}}\|\phi\|_{L^{1}}+C e^{-c t}\left\|\partial_{x}^{k} \phi\right\|_{L^{2}},
\end{aligned}
$$

where $0 \leq k \leq s+2$. These estimates can be obtained by (2.27) and (2.32). Here we omit the details.

When $M_{0}=\int_{\mathbb{R}}\left(u_{0}+u_{1}\right)(x) d x \neq 0$, we call

$$
\bar{v}(x, t)=M_{0} G_{0}(x, t+1)
$$


the diffusion wave with the amount $M_{0}$. Obviously, $\bar{v}(x, t)$ satisfies the following problem:

$$
\left\{\begin{array}{l}
\bar{v}_{t}-\beta \bar{v}_{x x}=0 \\
t=0: \quad \bar{v}=M_{0} G_{0}(x, 1) .
\end{array}\right.
$$

Therefore, $v-\bar{v}$ satisfies

$$
\left\{\begin{array}{l}
(v-\bar{v})_{t}-\beta(v-\bar{v})_{x x}=0 \\
t=0: \quad v-\bar{v}=u_{0}+u_{1}-M_{0} G_{0}(x, 1)
\end{array}\right.
$$

It is not difficult to prove the following lemma.

Lemma 4.2 Assume that $\int_{-\infty}^{x}\left(u_{0}(y)+u_{1}(y)-M_{0} G_{0}(y, 1)\right) d y \in L^{1}$, then

$$
\left\|\partial_{x}^{k}(v-\bar{v})(t)\right\|_{L^{2}} \leq C(1+t)^{-\frac{3}{4}-\frac{k}{2}}
$$

Combining Corollary 3.1, Lemma 4.1, and Lemma 4.2, we immediately have the following.

Theorem 4.1 Under the same assumption as Theorem 3.1, and also assuming that $M_{0}=$ $\int_{\mathbb{R}}\left(u_{0}+u_{1}\right)(x) d x \neq 0$ and $\int_{-\infty}^{x}\left(u_{0}(y)+u_{1}(y)-M_{0} G_{0}(y, 1)\right) d y \in L^{1}$, we let $u$ be the global solution to the problem (1.1), (1.2), which is constructed in Theorem 3.1 and we let $\bar{v}$ be the diffusion wave defined by (4.4). Then we have

$$
\left\|\partial_{x}^{k}(u-\bar{v})(t)\right\|_{L^{2}} \leq C(1+t)^{-\frac{3}{4}-\frac{k}{2}} .
$$

We have $M_{0}=\int_{-\infty}^{\infty}\left(u_{0}+u_{1}\right)(x) d x=0$ and $M_{1}=\int_{-\infty}^{\infty} U_{0}(x) d x \neq 0$, where $U_{0}(x):=$ $\int_{-\infty}^{x}\left(u_{0}+u_{1}\right)(y) d y$. We consider the initial value problem

$$
\left\{\begin{array}{l}
\rho_{t}-\beta \rho_{x x}=0 \\
t=0: \quad \rho=U_{0}(x)
\end{array}\right.
$$

and

$$
\left\{\begin{array}{l}
\bar{\rho}_{t}-\beta \bar{\rho}_{x x}=0, \\
t=0: \quad \bar{\rho}=M_{1} G_{0}(x, 1) .
\end{array}\right.
$$

When $\int_{-\infty}^{x}\left(U_{0}(y)-M_{0} G_{0}(y, 1)\right) d y \in L^{1}$, by applying Lemma 4.2, we have

$$
\left\|\partial_{x}^{k}(\rho-\bar{\rho})(t)\right\|_{L^{2}} \leq C(1+t)^{-\frac{3}{4}-\frac{k}{2}} .
$$

We call

$$
\tilde{v}(x, t)=M_{1} G_{0 x}(x, t+1)
$$

the diffusion wave with the amount $M_{1}$. Obviously, $v-\tilde{v}$ satisfies

$$
v-\tilde{v}=\partial_{x} \rho-\partial_{x} \bar{\rho} .
$$

From (4.11) and (4.13), we immediately obtain the following lemma. 
Lemma 4.3 Assume that $\int_{-\infty}^{x}\left(U_{0}(y)-M_{1} G_{0}(y, 1)\right) d y \in L^{1}$, then

$$
\left\|\partial_{x}^{k}(v-\tilde{v})(t)\right\|_{L^{2}} \leq C(1+t)^{-\frac{5}{4}-\frac{k}{2}}
$$

Corollary 3.1, Lemma 4.1, and Lemma 4.3 immediately give the following result.

Theorem 4.2 Under the same assumption as Theorem 3.1, also assuming that $M_{0}=$ $\int_{\mathbb{R}}\left(u_{0}+u_{1}\right)(x) d x=0, M_{1}=\int_{\mathbb{R}} U_{0}(x) d x \neq 0$, and $\int_{-\infty}^{x}\left(U_{0}(y)-M_{1} G_{0}(y, 1)\right) d y \in L^{1}$, where $U_{0}(x):=\int_{-\infty}^{x}\left(u_{0}+u_{1}\right)(y) d y$, we let $u$ be the global solution to the problem (1.1), (1.2), which is constructed in Theorem 3.1 and we let $\tilde{v}$ be the diffusion wave defined by (4.12). Then we have

$$
\left\|\partial_{x}^{k}(u-\tilde{v})(t)\right\|_{L^{2}} \leq C(1+t)^{-\frac{5}{4}-\frac{k}{2}} .
$$

\section{Competing interests}

The authors declare that they have no competing interests.

\section{Authors' contributions}

All authors contributed to each part of this work equally and read and approved the final manuscript.

\section{Author details}

${ }^{1}$ College of Electric Power, North China University of Water Resources and Electric Power, Zhengzhou, 450011, China. ${ }^{2}$ School of Mathematics and Information Sciences, North China University of Water Resources and Electric Power,

Zhengzhou, 450011, China.

Received: 26 January 2014 Accepted: 1 April 2014 Published: 17 Apr 2014

\section{References}

1. Takeda, H, Yoshikawa, S: On the initial value problem of the semilinear beam equation with weak damping I: smoothing effect. J. Math. Anal. Appl. 401, 244-258 (2013)

2. Takeda, H, Yoshikawa, S: On the initial data of the semilinear beam equation with weak damping II: asymptotic profiles. J. Differ. Equ. 253, 3061-3080 (2012)

3. Dai, W, Kong, D: Global existence and asymptotic behavior of classical solutions of quasilinear hyperbolic systems with linearly degenerate characteristic fields. J. Differ. Equ. 235, 127-165 (2007)

4. Kong, D, Yang, T: Asymptotic behavior of global classical solutions of quasilinear hyperbolic systems. Commun. Partial Differ. Equ. 28, 1203-1220 (2003)

5. Nakao, M, Ono, K: Existence of global solutions to the Cauchy problem for the semilinear dissipative wave equations. Math. Z. 214, 325-342 (1993)

6. Nishihara, K: Asymptotic behavior of solutions of quasilinear hyperbolic equations with damping. J. Differ. Equ. 137 384-395 (1997)

7. Ono, K: Global existence and asymptotic behavior of small solutions for semilinear dissipative wave equations. Discrete Contin. Dyn. Syst. 9, 651-662 (2003)

8. Takeda, H, Yoshikawa, S: Asymptotic profile of solutions for the limit unstable Cahn-Hilliard equation with inertial term. Differ. Integral Equ. 25, 341-362 (2012)

9. Geng, S: Convergence rates to asymptotic profile for solutions of quasilinear hyperbolic equations with linear damping. J. Hyperbolic Differ. Equ. 8, 115-129 (2011)

10. Wang, Y, Wei, Z: Global existence and asymptotic behavior of solutions to Cahn-Hilliard equation with inertial term Int. J. Math. 23(9), 1250087 (2012)

11. Yang, Z: Longtime behavior of the Kirchhoff type equation with strong damping on $\mathbb{R}^{n}$. J. Differ. Equ. 242, 269-286 (2007)

12. Sugitani, Y, Kawashima, S: Decay estimates of solution to a semi-linear dissipative plate equation. J. Hyperbolic Differ. Equ. 7, 471-501 (2010)

13. Wang, Y, Liu, F, Zhang, Y: Global existence and asymptotic of solutions for a semi-linear wave equation. J. Math. Anal. Appl. 385, 836-853 (2012)

14. Kato, M, Kawashima, S: Asymptotic behavior of solutions to the generalized cubic double dispersion equation in one space dimension. Kinet. Relat. Models 6, 969-987 (2013)

15. Wang, $\mathrm{S}, \mathrm{Xu}, \mathrm{H}$ : On the asymptotic behavior of solution for the generalized IBq equation with hydrodynamica damped term. J. Differ. Equ. 252, 4243-4258 (2012)

16. Zhang, Z, Zhang, Y: Global existence and asymptotic behavior of solutions to a class of fourth-order wave equations. Bound. Value Probl. 2013, 168 (2013)

17. Li, T, Chen, Y: Nonlinear Evolution Equations. Scientific Press, Beijing (1989) (in Chinese)

18. Zheng, S: Nonlinear Evolution Equations. Monographs and Surveys in Pure and Applied Mathematics, vol. 133. Chapman \& Hall/CRC, Boca Raton (2004) 
10.1186/1687-2770-2014-84

Cite this article as: Zhang and Li: Asymptotic profile of solutions to the semilinear beam equation. Boundary Value Problems 2014, 2014:84

Submit your manuscript to a SpringerOpen ${ }^{\circ}$ journal and benefit from:

- Convenient online submission

- Rigorous peer review

- Immediate publication on acceptance

- Open access: articles freely available online

- High visibility within the field

- Retaining the copyright to your article

Submit your next manuscript at $\gg$ springeropen.com 